\title{
Predisposição ao Alcoolismo: detecção via redes neurais e wavelets aplicadas ao ERP P300
}

\author{
Carla D. L. Becker \\ Universidade Federal de Pelotas - Centro de Engenharias \\ 96010-610, Pelotas, RS. E-mail: carla.diniz@ufpel.edu.br
}

\author{
Alice de J. Kozakevicius \\ Universidade Federal de Santa Maria - Departamento de Matemática \\ 97105-900, Santa Maria, RS. E-mail: alicek@ufsm.com
}

\section{Tiago Becker}

Universidade Federal do Rio Grande do Sul - Departamento de Engenharia Mecânica 90040-060, Porto Alegre, RS. E-mail: tiago_becker@ufrgs.br

\author{
Alberto A. Rasia-Filho \\ Universidade Federal de Ciências da Saúde de Porto Alegre - Departamento de Ciências Básicas \\ 90050-170, Porto Alegre, RS. E-mail: rasiafilho@pq.cnpq.br
}

\author{
Altamiro Amadeu Susin \\ Universidade Federal do Rio Grande do Sul - Departamento de Engenharia Elétrica \\ 90040-060, Porto Alegre, RS. E-mail: altamiro.susin@ufrgs.br
}

Resumo: Neste estudo propõe-se um método para classificar potenciais relacionados ao evento (ERP) P300, um tipo de sinal de eletroencefalograma (EEG), entre indivíduos com alto e baixo risco em desenvolver alcoolismo. A metodologia é composta por dois módulos: no primeiro é feita a aplicação da Transformada Wavelet Discreta (DWT) do sinal, que possibilita a extração de suas informações mais relevantes e no segundo, informações são processadas por uma rede neural artificial que identifica os dois padrões: alto e baixo risco. Após o treinamento, chegou-se a 89,47\% de classificação correta de sinais da base de dados considerada no Estudo Colaborativo sobre a Genética do Alcoolismo (COGA).

\section{INTRODUÇÃO}

O alcoolismo é uma doença crônica complexa, com consequências perigosas para a saúde humana [1]. Nos últimos anos, cerca de $3,8 \%$ de todas as mortes globais foram atribuídas ao abuso de álcool, 6,2\% em homens e 1,1\% nas mulheres [1]. Fatores genéticos e psicossociais influenciam o desenvolvimento e as manifestações clínicas do alcoolismo [2-6], como apontado pelo grupo de pesquisa americano "Estudo Colaborativo sobre a Genética do Alcoolismo" (COGA), que entre outras técnicas, considera sinais cerebrais em suas análises.

Um conceito importante explorado por este grupo é com relação à resposta evocada ou potencial relacionado a um evento (ERP), que são as respostas do sistema nervoso central (SNC) a estímulos sensoriais ou elétricos. O ERP é um tipo de sinal de eletroencefalograma (EEG), isto é, uma série de ondas positivas e negativas que são geradas acima do tronco cerebral e com extensa distribuição no escalpo e com latência que varia de 250-600 ms [2].

O ERP P300 é obtido quando o paciente está acordado, alerta e distingue um estímulo combinado entre um grupo de estímulos, representando o período de resposta do córtex cerebral de associação ao estímulo do córtex primário [2-6]. Os resultados das análises do COGA mostraram que o ERP P300 tem amplitude significativamente mais fraca em indivíduos com alto risco (AR) em desenvolver o alcoolismo, do que em indivíduos com baixo risco (BR) [2-6]. Mais recentemente, verificou-se que as ondas cerebrais delta $(1-3 \mathrm{~Hz})$ e teta $(3,5-7,5 \mathrm{~Hz})$, que são as principais componentes dos sinais ERP P300, são reduzidas em alcoolistas e indivíduos com predisposição ao alcoolismo [2-6]. Estas ondas possuem uma relação mais próxima e 
profunda com o SNC e com a função ligada à vulnerabilidade ao alcoolismo, tornando-se um endofenótipo mais estável do que o próprio sinal P300 [2-6].

As diversas análises realizadas pelo grupo COGA diferenciam sinais de EEG e ERP de indivíduos com AR e BR para o alcoolismo, comparando por meio da análise de variância (ANOVA) a amplitude máxima dos sinais ERP P300 [2-4] e a energia por faixa de frequência do sinal de EEG com base na transformada de Fourier (TF) [5-6]. Os estudos do COGA realizados de 1999 a 2012 [2-6] encontraram uma redução da amplitude de potenciais visuais em indivíduos com predisposição para o alcoolismo, quando um estímulo bem diferenciado é inserido em uma tarefa de discriminação visual. Já na metodologia proposta neste estudo, para a extração de características dos sinais P300, é considerada a transformada wavelet discreta (TWD), cuja base são funções ortonormais de família de Daubechies. Como os sinais analisados são de natureza não-estacionária e não-periódica, a aplicação de transformadas wavelets torna-se uma ferramenta poderosa para extração de informações complementares destes sinais, contribuindo para o estudo e a compreensão das características que diferenciam os sinais de EEG e ERP de indivíduos com AR e BR para desenvolver alcoolismo.

Após a seleção das informações mais relevantes em cada sinal com base na TWD, na segunda etapa deste trabalho, um classificador neural do tipo "perceptron" multicamadas (MLP) treinado com o algoritmo "backpropagation", é proposto para classificar a diferença funcional entre os grupos de indivíduos com AR e BR. Além de compreender a natureza da onda ERP P300 e como suas componentes podem ser utilizadas para diferenciar os indivíduos com AR e BR, o objetivo deste trabalho é também identificar quais as melhores regiões, ou posições do cérebro, cujos dados sejam mais propícios para este tipo de análise.

\section{BANCO DE SINAIS}

Neste trabalho é utilizada uma das bases de sinais de potenciais visuais de indivíduos com AR e BR disponibilizada pelo grupo COGA, cedida pelo Dr. Ingber do "Neurodynamics Laboratory at the State University of New York Health Center" em 1999 [7]. Estes dados foram estudados pelos autores no período de 2004 a 2010 [8], período anterior a Resolução CNS N ${ }^{\circ}$ 441/11, sendo assim, não foi necessária aprovação do estudo pelo Comitê de Ética da UFRGS.

Para a captação do sinal cada indivíduo usou 64 eletrodos alocados nas posições padronizadas para os exames de EEG. Todos os indivíduos eram destros, com visão normal ou corrigida e não apresentavam desordens psiquiátricas. Não houve qualquer diferença de gênero observada nos resultados dos sinais analisados [7]. O objetivo do experimento era o de obter o ERP P300, que é extraído quando os indivíduos estão engajados em uma tarefa visual que requer atenção. Os estímulos eram compostos por imagens de objetos. Todas as imagens selecionadas representavam diferentes objetos reais e foram facilmente identificadas pelos participantes do experimento. As figuras eram mostradas aos participantes de duas formas: (1) Visualizar um único objeto; (2)Visualizar dois objetos: um objeto S1 e um objeto S2, mostrados em duas condições: "matching" (Ma) e "nonmatching" (Nma).

$\mathrm{Na}$ condição $\mathrm{Ma}$, a primeira figura $\mathrm{S} 1$ era idêntica à segunda figura S2. Já na condição Nma, a figura $\mathrm{S} 1$ era seguida por uma outra figura $\mathrm{S} 2$, completamente diferente da primeira $\mathrm{S} 1$, em termos de sua categoria semântica. A apresentação das tarefas Ma e Nma foi aleatória. Em metade das tarefas o estímulo teste S2 era idêntico a S1, e na outra metade das tarefas, o estímulo teste S2 era diferente do S1. A tarefa do indivíduo era decidir se a figura S2 apresentada a ele era a mesma do primeiro estímulo S1. Cada indivíduo respondeu em média a 120 tarefas [7].

\section{A. Extração dos potenciais P300}

Para cada um dos 38 indivíduos selecionados do banco [7], extraíram-se os ERPs para a condição Ma e Nma. Para a extração dos ERPs, realiza-se a média dos sinais de EEG obtidos do indivíduo respondendo a um estímulo específico (neste caso, Ma e Nma), como executado nos trabalhos no COGA [2-7]. Para exemplificar a forma do sinal de ERP extraído para cada condição e indivíduo, na Figura 1 é apresentada a média de sinais ERP Ma e Nma do eletrodo 
PO2 da região posterior do escalpo, dos indivíduos com BR à esquerda e dos indivíduos com AR à direita. As ANOVAs realizadas nos dados de 1999 a 2012 [2-7] mostraram que existem diferenças significativas $(p>0.001)$ na amplitude do potencial ERP P300, ao comparar as condições Nma e Ma, principalmente na janela de 250-600 ms, entre os grupos BR e AR.
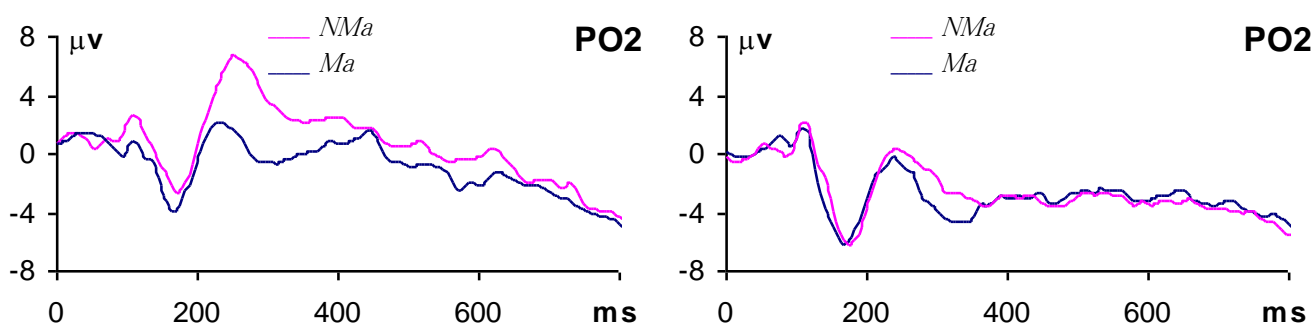

Figura 1: Média dos Sinais de ERP da condição Ma e Nma do eletrodo PO2 dos indivíduos com e BR (esquerda) e AR (direita).

\section{TRANSFORMADA WAVELET}

Uma das principais diferenças entre as diversas pesquisas apresentadas pelo grupo COGA e este trabalho, está na análise baseada em wavelets, ao invés da análise baseada na TF [5-6].

Convencionalmente o processo de converter um sinal no domínio do tempo para o domínio da frequência é obtido por meio da FT, porém esta transformada não fornece informação suficiente quando se trata de sinais não-estacionários, como os sinais de EEG e ERP [10]. A FT determina as componentes de frequência de um sinal, mas não determina onde esta frequência está localizada no tempo. Já a TW permite a análise com janelas de múltipla duração, o que permite uma perspectiva de análise em multiresolução do sinal.

Neste trabalho, para a obtenção da TWD são consideradas wavelets ortonormais da família de Daubechies [16], como base da transformada a ser considerada no primeiro módulo do esquema de análise. As wavelets utilizadas neste processo são a $\mathrm{Db} 2$, com dois momentos nulos (4 filtros) e a Db6 com 6 momentos nulos (12 filtros), sendo o número de momentos nulos um indicador de suavidade da função. Estas funções vêm sendo aplicadas na decomposição e localização de distúrbios e faltas em vários tipos de sinais, dentre eles: linhas em sistemas de potência [9] e em sinais de EEG para estudos sobre epilepsia [11]. A TWD possui uma formulação rápida envolvendo apenas os filtros das funções base (algoritmo de cascata), proposta por Mallat [16]. Para sinais não periódicos é considerada a formulação periodizada da TWD [17]. Assim, dado um sinal de entrada $\mathrm{A}_{0}, \operatorname{com} N_{0}=2^{J}$ pontos, o primeiro nível da TWD decompõe este sinal em dois conjuntos (vetores) de coeficientes: $\mathrm{A}_{l}$ e $D_{l}$, ambos com $N_{l}=2^{(J-l)}$ elementos. No nível seguinte de decomposição, apenas o vetor de coeficientes de escala, $\mathrm{A}_{l}$, é decomposto em dois novos vetores $\mathrm{A}_{2}$ e $D_{2}$, ficando inalterado o conjunto de coeficientes wavelets $D_{l}$. Seguindo este processo, um sinal inicial $\mathrm{A}_{0}$ pode ser decomposto em $k$ níveis de resolução, gerando a seguinte sequência de vetores contendo os coeficientes de cada nível $\left(A_{k}\right.$, $\left.D_{k}, D_{k-1}, \ldots, D_{2}, D_{l}\right)$. Através da TWD inversa o sinal original é reconstruído exatamente a partir desta fatoração em $k$ níveis.

Através da TWD é possível ressaltar e capturar de forma eficaz as informações do potencial evocado visual P300 de cada um dos sinais analisados, através da análise de seus coeficientes wavelets.

\section{A. Operação de limiarização}

Identificada a escala (faixa de frequência), onde estão caracterizadas as diferenças mais significativas entre os sinais dos grupos AR e BR, pode-se alimentar um classificador neural com os CW significativos que representam essas diferenças mais relevantes entre os dois grupos, possibilitando assim que a rede reconheça os padrões existentes de forma mais eficiente. Para que os CW mais significativos possam ser identificados e selecionados, é aplicada uma regra de limiarização em todos os níveis da TWD. De acordo com a limiarização escolhida, os CW acima do limiar de corte $\lambda_{j}$ (significativos) são preservados de forma 
inalterada, enquanto aqueles menores do que $\lambda_{j}$ são zerados e portanto, descartados da segunda etapa de análise, como dado pela expressão (1).

$$
\operatorname{Trh}\left(d_{j, k}\right)=\left\{\begin{array}{l}
d_{j, k}, \text { if }\left|d_{j, k}\right|>\lambda_{j} \\
0, \text { if }\left|d_{j, k}\right| \leq \lambda_{j}
\end{array}\right.
$$

Três foram os limiares aplicados aos coeficientes de detalhe de cada nível $k$ da TWD, a saber: a média, a energia e o limiar de corte universal proposto por Donoho e Johnstone [12]. Para uma dada escala $j$, e um certo nível $k$ com um número $N$ de $\mathrm{CW}$ de detalhe $d$, o limiar de corte em [12] é calculado pela expressão (2):

$$
\lambda_{k}=\sigma \sqrt{2 \log _{10}(N)}
$$

sendo $\sigma$ que representa a estimativa do ruído e é dado por: $\sigma=$ mediana $\left(\left|d_{j}\right|\right) / 0,6745$.

\section{REDES NEURIAS ARTIFICIAIS}

Uma Rede Neural Artificial (RNA) é um modelo matemático e computacional não-linear para o processamento de informações, com arquiteturas inspiradas pela organização neuronal [13-14]. As RNAs são utilizadas com sucesso como ferramenta auxiliar no diagnóstico de crises epilépticas [11] e de detecção de potenciais evocados [15], para mencionar apenas algumas aplicações biomédicas possíveis.

As RNAs implementadas neste trabalho baseiam-se na técnica de redes do tipo MLP com o algoritmo de treinamento "backpropagation", uma vez que o problema tratado neste estudo é basicamente uma classificação de amostras. A camada de saída das RNAs possuem dois neurônios. De acordo com a matriz criada, se o maior valor for obtido pelo neurônio 1, o indivíduo é classificado como AR. Já se o maior valor for obtido agora pelo neurônio 2, o indivíduo é classificado como do grupo BR. A dimensão do vetor de entrada $\left(x_{1}, x_{2}, x_{3}, \ldots, x_{n}\right)$ das RNAs variou de acordo com o número de $\mathrm{CW}$ aplicados na entrada da rede. A função de ativação utilizada na saída do MLP é a tangente hiperbólica.

Se o objetivo do classificador neural é o de uma investigação completa sobre a variação do modelo em relação aos dados utilizados, como é o caso deste estudo, pode-se usar a forma extrema de validação cruzada (CV) múltipla, conhecida como o "leave-one-out method" [13]. Neste caso, $N$ - 1 exemplos são usados para treinar o modelo, e o modelo é validado testando-o sobre o exemplo deixado de fora. O experimento é repetido $\mathrm{N}$ vezes, cada vez deixando-se de fora um exemplo diferente para a validação. Sendo assim, na implementação desta etapa do trabalho, o arquivo com os dados do grupo BR foi dividido em 19 arquivos, assim como o arquivo do grupo de AR. Com os arquivos divididos desta forma, treina-se a rede com a união de 36 arquivos (18 do grupo de BR e 18 do grupo de AR) e testa-se o desempenho com o arquivo restante, ou seja, um do grupo de BR e outro de AR. Este processo é repetido dezenove vezes de forma que o arquivo de teste é trocado a cada CV. O erro médio quadrático (MSE) na validação é então a média sobre as $N$ tentativas do experimento [13]. Neste trabalho, utiliza-se o "Toolbox" de Redes Neurais do Matlab®, que contém várias funções parametrizadas que permitem a prototipação com redes diretas.

\section{METODOLOGIA PROPOSTA}

A metodologia desenvolvida neste trabalho tem por objetivo quantificar a diferença da resposta em amplitude do ERP P300 entre as condições Nma e Ma nos indivíduos com BR e com AR. Diferentemente dos trabalhos do COGA [2-6], neste trabalho a distinção entre os grupos com AR e com BR é executada com base na variação relativa da diferença entre a condição Ma e a condição Nma de um grupo para outro. No grupo de BR, esta variação é percentualmente maior. Porém, de um indivíduo para outro (independente do grupo), a amplitude dos sinais de ERP varia, de forma que a comparação da diferença relativa não pode ser feita diretamente no valor medido dos sinais. 
Por esta razão, o procedimento adotado para fazer a comparação é subtrair o sinal Ma do Nma de cada indivíduo, considerar para cada posição o valor absoluto desta operação e dividílo pelo "root mean square" (RMS) do sinal Ma, gerando assim, $A_{0}$.

O sinal de entrada $A_{0}$ é inicialmente transformado pela TWD em $k=7$ níveis e limiarizado pela expressão (1), gerando $A_{7}, \widetilde{D}_{7}, \ldots, \widetilde{D}_{4}, \widetilde{D}_{3}, \widetilde{D}_{2}, \widetilde{D}_{1}$. Os CW significativos $A_{7}, \widetilde{D}_{7}, \widetilde{D}_{4}, \ldots, \tilde{D}_{3}, \tilde{D}_{2}, \tilde{D}_{1}$, são, então, passados para as RNAs, que têm por objetivo classificar dois tipos de dados:

*Os CW obtidos do sinal formado pela diferença relativa entre os ERPs Nma e Ma de indivíduos com AR;

*Os CW obtidos do sinal formado pela diferença relativa entre os ERPs Nma e Ma de indivíduos com BR.

O desempenho de classificação das RNAs implementadas é obtido pela acurácia (\%), definida como o quociente entre as amostras de AR e BR que foram corretamente classificadas e o total de amostras.

\section{A.Classificação do potencial P300 através das RNAs}

Neste trabalho são analisadas três configurações de RNAs, alimentadas com coeficientes de aproximação (A) e de detalhes restantes após o processo de limiarização $(\tilde{D})$. Apenas os resultados para o limiar de Donoho serão apresentados, pelo fato da taxa de acerto na classificação ter sido maior. Na tabela 1 são apresentadas as configurações das RNAs implementadas a para TWD com Db2 como função base, em três níveis de decomposição distintos: $A_{5}+\tilde{D}_{5}, A_{5}+\widetilde{D}_{5}+\widetilde{D}_{4} e A_{5}+\widetilde{D}_{5}+\widetilde{D}_{4}+\tilde{D}_{3}$, a dimensão máxima da camada de entrada (DMCE), o número máximo de neurônios na camada oculta (NNCO), o espectro de frequência (EF) presente em cada decomposição e a oscilação cerebral (OC).

\begin{tabular}{cccc}
\hline \multicolumn{4}{c}{ RNAs Implementadas com Db2 } \\
\hline \multirow{2}{*}{ EF } & $\begin{array}{c}A_{5}+\tilde{D}_{5} \\
(0-5,34 \mathrm{~Hz})\end{array}$ & $\begin{array}{c}A_{5}+\tilde{D}_{5}+\tilde{D}_{4} \\
(0-10,66 \mathrm{~Hz})\end{array}$ & $\begin{array}{c}A_{5}+\tilde{D}_{5}+\tilde{D}_{4}+\tilde{D}_{3} \\
(0-21,34 \mathrm{~Hz})\end{array}$ \\
\hline OC & Delta & Delta e teta & Delta, Teta, Alfa e Beta \\
\hline RNA & RNA1 & RNA2 & RNA3 \\
\hline DMCE & 20 & 38 & 72 \\
\hline NNCO & 8 & 12 & 20 \\
\hline \multicolumn{2}{l}{ Tabela1: Dados gerais das configurações das RNAs implementadas com CW Db2. }
\end{tabular}

Construiu-se uma RNA para cada um dos 22 eletrodos que captam os sinais de EEG da região posterior do cérebro (parietal, occipital, temporal esquerda e temporal direita), pois é nesta região que se observa a maior diferença em amplitude no potencial P300 [2] (dado também observado na etapa de extração dos ERPs dos grupos indivíduos com AR e BR desta pesquisa, como visto na Figura 1). Assim, após a análise dos resultados de classificação, obtémse um "mapa" de localização no escalpo onde a diferenciação do processamento de informação dos dois grupos analisados é mais relevante.

O número de entradas da rede afeta o número de iterações necessárias para a rede encontrar os valores das conexões entre os neurônios. Para cada configuração, o treinamento começa com um pequeno número de neurônios na camada oculta $(\mathrm{CO})$. Este número é aumentado até atingir-se os melhores resultados de classificação. Os parâmetros e as estruturas das RNAs são selecionados para se obter o melhor desempenho, após várias experiências. Outra diferença entre as RNAs implementadas está na dimensão da camada de entrada (CE), dependente do número de $\mathrm{CW}$, mas para todas foi estipulado um MSE a ser alcançado na ordem de $10^{-10}$. Todas as redes são treinadas até que o MSE seja alcançado ou até completar o número de épocas de treinamento.

Pretende-se com estas configurações de RNAS identificar os CW dos níveis que contêm frequências delta e teta, ou seja, os CW mais significativos na formação ERP P300, que levem a resultados corretos de classificação das RNAs, avaliando também, a contribuição de $\mathrm{CW}$ de outros níveis de decomposição, que contém as informações sobre a frequência alfa $(8-12,5 \mathrm{~Hz})$ 
e beta $(13-28,5 \mathrm{~Hz})$, isto é, se estes CW também apresentam informações relevantes para formação do sinal de ERP, melhorando o desempenho da classificação dos RNAs.

\section{RESULTADOS}

Os sinais dos eletrodos $\mathrm{P} 3, \mathrm{P} 4, \mathrm{P} 8, \mathrm{PO} 2, \mathrm{PO} 7$ e OZ classificados com os $\mathrm{CW}$ oriundos da wavelet $\mathrm{Db} 2$ e limiarizados de acordo o corte universal proposto em [12], são os que obtiveram o melhor índice de classificação. Para a RNA1, a taxa de sucesso na classificação atingido para estes eletrodos é de $86,87 \%$. Nas configurações RNA2 e RNA3, onde os coeficientes de detalhe $(\mathrm{CW})$ de outros níveis são incorporados à entrada das RNAs, os resultados de classificação das redes decaem, como pode ser visto na Tabela 2.

\begin{tabular}{ccccccccccccc}
\hline & Eletrodo & P1 & P2 & P3 & P4 & P5 & P6 & P7 & P8 & PZ & PO1 & PO2 \\
\cline { 2 - 11 } & RNA1 & 78,94 & 73,68 & 86,84 & 86,84 & 71,05 & 81,15 & 81,15 & 86,84 & 71,05 & 81,15 & 86,84 \\
\cline { 2 - 11 } & RNA2 & 73,68 & 76,31 & 73,68 & 73,68 & 73,68 & 78,94 & 78,94 & 76,51 & 73,68 & 76,51 & 78,94 \\
\cline { 2 - 11 } $\begin{array}{c}\text { Acurácia } \\
(\%)\end{array}$ & RNA3 & 71,05 & 73,68 & 71,05 & 71,05 & 71,05 & 73,68 & 76,51 & 73,68 & 73,68 & 73,68 & 73,68 \\
\cline { 2 - 11 } & Eletrodo & PO7 & PO8 & POZ & O1 & O2 & OZ & CP1 & CP2 & CP3 & CP4 & CPZ \\
\cline { 2 - 11 } & RNA1 & 86,84 & 76,51 & 78,94 & 76,51 & 76,51 & 86,84 & 71,05 & 68,42 & 71,05 & 65,75 & 68,42 \\
\cline { 2 - 11 } & RNA2 & 73,68 & 76,51 & 76,51 & 81,15 & 81,15 & 78,94 & 73,68 & 68,42 & 76,51 & 71,05 & 73,68 \\
\cline { 2 - 10 } & RNA 3 & 71,05 & 63,15 & 71,05 & 76,51 & 71,05 & 73,68 & 68,42 & 68,42 & 68,42 & 57,89 & 63,15 \\
\hline
\end{tabular}

Tabela 2: Resultados de classificação obtidos com os coeficientes da wavelet Db2.

Construiu-se, então, RNAs para os sinais dos eletrodos com os melhores resultados de classificação, com os CW da TWD Db6, para validar a metodologia de classificação proposta, em duas configurações. A primeira RNA contêm coeficientes do nível de aproximação $\mathrm{A}_{4} \mathrm{e}$ a segunda RNA contêm os CW $A_{5}+\widetilde{D}_{5}$, ambas com conteúdo espectral $(0-5,81 \mathrm{~Hz})$. A Tabela 3 apresenta os resultados de classificação obtidos para estas RNAs. Os resultados de classificação obtidos com os CW Db6, mostram uma taxa de acerto de 89,47\% confirmando então, o potencial da metodologia proposta para classificação de sinais com AR e BR.

\begin{tabular}{ccccccccc}
\hline \multirow{2}{*}{$\begin{array}{c}\text { Acurácia } \\
(\%)\end{array}$} & Eletrodo & P3 & P4 & P8 & PO1 & PO2 & PO7 & OZ \\
\cline { 2 - 9 } & RNA1 & $\mathbf{8 9 , 4 7}$ & $\mathbf{8 9 , 4 7}$ & 84,21 & $\mathbf{8 9 , 4 7}$ & 86,84 & 86,84 & 84,21 \\
\cline { 2 - 9 } & RNA2 & 86,84 & 86,84 & 86,84 & $\mathbf{8 9 , 4 7}$ & 84,21 & 86,84 & 86,84 \\
\hline
\end{tabular}

Tabela 3. Resultados de classificação obtidos com os coeficientes da wavelet Db6.

\section{CONCLUSÃO}

Em um estudo anterior [9] o ERP, componente C247, que reflete o processo de reconhecimento, de indivíduos com AR e BR para o alcoolismo, foi investigado. Os resultados sugeriram que a amplitude do C247 para a condição Ma foi significativamente diminuída em comparação com NMa para o grupo com BR, mas o mesmo não foi observado no grupo de AR. O uso das RNAs e da DWT Lemarie foi utilizado para a classificação do C247. As redes com coeficientes Lemarie foram capazes de classificar corretamente aproximadamente $71 \%$ dos sinais de ERP dos dois grupos. Observa-se que a diferença relativa obtida das condições NMa e Ma, e a utilização da DWT de Daubechies no presente trabalho, permitiram melhorar a técnica de identificação de AR e BR indivíduos (aproximadamente 90\%) usando um número reduzido de coeficientes wavelets, em comparação com ao estudo passado (ver detalhes em [9]).

Com estes resultados, conclui-se que a onda delta é realmente a oscilação preponderante no ERP P300 e, que os CW com conteúdo espectral da frequência delta, obtidos através da decomposição do sinal formado pela diferença entre os sinais NMa e Ma caracterizam e diferenciam os grupos com AR e BR em desenvolver o alcoolismo, além de caracterizar os eletrodos P3, P4, P8, PO1, PO2, PO7 e OZ (que correspondem a regiões do córtex cerebral envolvidas no processamento associativo e multimodal das informações percebidas, o que é 
atividade necessária para percepção plena, interpretação, julgamento e execução das tarefas que os pacientes avaliados executaram) como os mais significativos nesta análise.

\section{Agradecimentos}

Ao Dr. Lester Ingber, do "Neurodynamics Laboratory at the State University of New York Health Center", pela cedência [7] dos registros eletroencefalográficos utilizados nesta pesquisa.

\section{Referências}

[1] Global Health Observatory (GHO), (Acesso em: 21 de Junho de 2013). Disponível em: http://www.who.int/gho/alcohol/harms_consequences/deaths_alcohol/en/.

[2] M. Rangaswamy, K. A. Jones, B. Porjesz, D. B. Chorlian, A. Padmanabhapillai, C. Kamaraja, S. Kuperman, J. Rohrbaugh, S. J. O'Connor, L. O. Bauer, M. A. Schuckit, H. Begleiter, Delta and theta oscillations as risk markers in adolescent offspring of alcoholics, International Journal of Psychophysiology, vol. 63, no 1, pp. 305, (2007).

[3] C. Kamarajam, B. Porjesz, K. Jones, D. Chorlian, A. Padmanabhapillai, M. Rangaswamy, A. Stimus, H. Begleiter, Event-Related oscillations in offspring of alcoholics: Neurocognitive Disinhibition as a Risk for Alcoholism, Biological Psychiatry, vol. 59, no 7, pp. 625-634, (2006).

[4] C. Kamarajan, M. Rangaswamy, N. Manz, D. B. Chorlian, A. K. Pandey, B. N. Roopesh, B. Porjesz, Topography, Power, and Current Source Density of Theta Oscillations During Reward Processing as Markers for Alcohol Dependence, Human Brain Mapping, vol. 33, no 5, pp. 1019-1039, (2012).

[5] K. A. Jones, B. Porjesz, D. Chorlian, M. Rangaswamy, C. Kamarajan, A. Padmanabhapillai, A. Stimus, H. Begleiter, "S-transform time-frequency analysis of P300 reveals deficits in individuals diagnosed with alcoholism", Clin. Neurophysiol.; vol. 117, no 10, pp. 2128-2143, (2006).

[6] A. K Pandey, C. Kamarajan, M. Rangaswamy and B. Porjesz, Event-Related Oscillations in Alcoholism Research: A Review, Journal of Addiction Research \& Therapy, S7:001, (2012).

[7] L. Ingber, (Acesso em: 07 de Abril de 2014). Disponível em: http://kdd.ics.uci.edu/databases/eeg/eeg.html

[8] C. D. Lopes, "Um Método de Avaliação da Amplitude do Potencial P300 Comparando Indivíduos com Alto Risco e Baixo Risxo para o Alcoolismo", Tese de Doutorado, PPGEEUFRGS, 2010.

[9] C. D. Lopes, J. O. Mainardi, M. A Zaro, A. A. Susin, Classification of Event-Related Potentials in Individuals at Risk for Alcoholism Using Wavelet Transform and Artificial Neural Network, IEEE 2004 Symposium on Computational Intelligence in Bioinformatics and Computational Biology (CIBCB), La Jolla, pp. 123-128, (2004).

[10] E. F. Arruda, et al. "Um estudo das famílias wavelets aplicadas à qualidade da energia elétrica". In: Congresso Brasileiro de Automática, CBA, Natal, RN: CBA, 6p, (2002).

[11] R. Panda, P. S. Khobragade, P. D. Jambhule, S. N. Jengthe, P. R. Pal, T. K. Gandhi, Classification of EEG signal using wavelet transform and support vector machine for epileptic seizure diction, Systems in Medicine and Biology (ICSMB), pp. 405-408, (2010).

[12] D. L Donoho, I. M Johnstone, Ideal spatial adaptation via wavelet shrinkage, Biometrika, [S. 1], vol. 81, no 3, pp. 425-455, (1994).

[13] S. Haykin, "Neural Networks: A comprehensive foundation", Mcmillan, 1994.

[14] I. Omerhodzic, S. Avdakovic, A. Nuhanovic, K. Dizdarevic, Energy Distribution of EEG Signals: EEG Signal Wavelet-Neural Network Classifier, International Journal of Biological and Life Sciences, vol. 6, no 4, (2010).

[15] A. P. Souza, A. M. F. L. Miranda de Sá, E. M. A. M. Mendes, C. J. Tierra-Criollo and L. B. Felix., Enhancing Visual Evoked Potentials Detection with Use of Computational Intelligence Tools, International Journal of Biomedical Data Mining, vol. 1, (2011).

[16] I. Daubechies, "Ten Lectures on Wavelets", SIAM, 1992.

[17]S. Mallat, "A Wavelet Tour of Signal Processing”, Academic Press, 2008. 